\title{
A BEM and FEM analysis of fluid-structure interaction in a double tank
}

\author{
J. Ravnik ${ }^{1}$, E. Strelnikova ${ }^{2}$, V. Gnitko ${ }^{2}$ \& U. Ogorodnyk ${ }^{2}$ \\ ${ }^{1}$ Faculty of Mechanical Engineering, University of Maribor, Slovenia \\ ${ }^{2}$ A. N. Podgorny Institute for Mechanical Engineering Problems, \\ Ukrainian Academy of Sciences, Ukraine
}

\begin{abstract}
In this paper we present a fluid-structure interaction analysis of shell structures with compartments partially filled with a liquid. The compound shell was a simplified model of a fuel tank. The shell is considered to be thin and Kirghoff-Lave linear theory hypotheses are applied. The liquid is ideal and incompressible. Its properties and the filling levels may be different in each compartment. The coupled problem is solved using a coupled BEM and FEM in-house solver. The tank structure is modelled by FEM and the liquid sloshing in fluid domain is described by BEM. The method relies on determining the fluid pressure from the system of singular integral equations. For its numerical solution the boundary element method was applied. The boundary of the liquid computational domain is discretized by nine-node boundary elements. The quadratic interpolation of functions and linear interpolation of flux are involved. The natural frequencies were obtained for the cylindrical double tank with two compartments.
\end{abstract}

Keywords: fluid-structure interaction, baffles, free vibrations, boundary and finite element methods.

\section{Introduction}

Different engineering fields such as the aerospace and chemical industry, power machine building, wind power engineering and transport extensively use thin-wall structural elements operated under excess process loads. In many circumstances 
these shells and shell structures are subjected not only to static loads but also to dynamic disturbances and filled with internal fluid. The influences of both shell and fluid on each other must not be neglected in stress-strength analysis of these structural elements. So the interaction between the sloshing liquid and the shell structure has been the challenging field of research in many engineering applications.

Liquid sloshing is an interesting physical phenomenon of enormous practical interest that has far reaching applications in a wide field of technologies and engineering disciplines. It occurs in moving tanks with contained liquid masses such as rocket tanks, marine and space vehicles as well as in seismically excited storage tanks, dams, reactors, and nuclear vessels [1].

Many different types of model tests at different scales and with different objectives were proposed and performed in the last few years in this research area.

Since the launch of the early high-efficiency rockets in 1957, controlling liquid fuel slosh within a launch vehicle has been a major design concern. Moreover, with today's large and complex spacecraft, a substantial mass of fuel is necessary to place them into orbit and to perform orbital maneuvers. The mass of fuel contained in the tanks of a geosynchronous satellite amounts to approximately $40 \%$ of its total mass [2]. When the fuel tanks are only partially filled, large quantities of fuel move inside the tanks under translational and rotational accelerations and generate the fuel slosh dynamics. Slosh control of propellant is a significant challenge to spacecraft stability. Mission failure has been attributed to slosh-induced instabilities in several cases [3, 4].

As the propellant level decreases throughout a mission, the effects of sloshing forces on the remaining fuel become more prominent. When the tank is full or nearly so, the fuel lacks the open space to slosh. However in the latter stages of the mission, when most of the fuel has been consumed, the fuel has sufficient volume to slosh and possibly disturb the flight trajectory. This sloshing can ultimately lead to wobble in a spinning spacecraft and self-amplifying oscillations that can result in failure of individual instruments or failure of the entire structure. The dynamics of a fluid that interacts with the walls of its container are complicated and challenging to predict. The effects of sloshing on bodies in motion are significant and in some cases devastating. These effects remain prominent even when the propellant volume represents only $0.3 \%$ of the total spacecraft mass [5].

In order to suppress sloshing a variety of methods have been proposed, simulated and tested. The effects of baffle on sloshing frequency have been studied in [6]. The mathematical technique used here is based on the velocity potential function; the problem was solved using finite-element analysis.

The motion of liquid within a partially filled tank in [7] was investigated by representing the fluid slosh through an equivalent mechanical system using a pendulum analogy model. The model parameters were computed based on inviscid fluid flow conditions and the dynamic fluid slosh forces arising due to the dynamics of the vehicle during a given maneuver were computed using the equivalent mechanical system. 
Liquid sloshing in partially filled horizontal cylindrical tanks with circular cross sections is a common problem in the road transportation industry that has been extensively studied for many years [8-10]. A recent review on liquid sloshing in horizontal cylindrical tanks was accomplished in [11].

In order to restrain the fluid sloshing motion a common technique is to place additional sub-structures called baffles or separators within the tank [8].

So we have the problem of sloshing in so-called double tanks or tanks with the compartments.

The issue of suppression of sloshing behavior using baffles goes back to late $50 \mathrm{~s}$ when lots of experimental and theoretical studies were concerned with the effect of baffles on the sloshing in fuel containers of space vehicles $[12,13]$.

Since then, numerous authors have tackled the subject. Strandberg in [8] performed an experimental investigation on dynamic performance and stability of horizontal circular tank. He also studied the overturning limit for half-full elliptical containers with various baffle configurations and concluded that the vertical baffle must be preferred in comparison with the un-baffled or horizontally baffled elliptical container. In [14] authors used the method of eigenfunction series to explore the effect of a thin vertical baffle in a fluid-filled rectangular tank on fluid frequencies. In [15] this technique was subsequently extended on circular containers having internal baffles. In [16] a mathematical model was developed for the ship rolling motion with free surface liquids on board; numerical and experimental results for a rectangular tank with a vertical bottom-mounted internal baffle were presented. In [17] the authors used the FLUENT software to develop a three-dimensional nonlinear model of a partly-filled cylindrical tank with and without baffles to investigate the significance of resulting destabilizing forces and moments. The main objective in [2] was to analyze multi-excitation effects on a cylinder divided by plate on two compartment on the base pf BEM and FEM numerical analysis. Diverse multi-exciting forces were applied on this base plate with different frequencies whereas, independently calculated results were superimposed to provide consolidated result.

The above review clearly indicates that there exists a massive body of literature on liquid sloshing in rectangular or upright cylindrical containers with various baffle configurations. With respect to all the numerical work which has been done, it is fair to say that there is still no fully efficient numerical method to deal with the sloshing in fluid-structure interactions in two-compartment tanks. Indeed it appears that, from computational point of view, it is impossible to account for all the different physical effects at the same time.

In this work we proposed the method of fluid-structure interaction analysis for tanks with compartments partially filled with liquids that allows us to include elasticity of shell walls, different liquid properties in each compartment, gravity force and to estimate influence of these factors on frequencies of tank vibration.

\section{Problem statement}

Let us consider the coupled problem for shell structure with two compartments partially filled with the liquid (Fig. 1). In this study we consider the cylindrical 
shell with bottom and baffle. The contained liquid is assumed to be inviscid and incompressible. We suppose that liquid properties and filling levels may be different in each compartment.
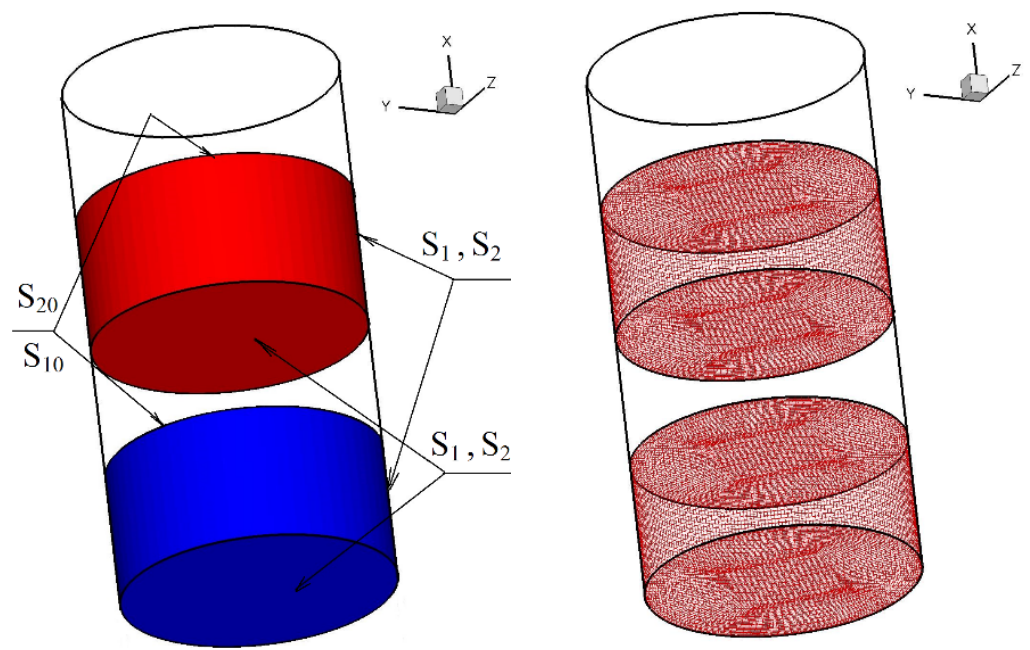

Figure 1: Fluid-filled double tank.

Suppose that the flow induced by vibrations of the shell is irrotational and consider small shell and fluid vibrations. Let $\boldsymbol{U}$ is the vector-function of the shell displacements. A system of governing equations of motion of elastic shell with the liquid in the operator form is given by

$$
\boldsymbol{L} \boldsymbol{U}+\boldsymbol{M} \ddot{\boldsymbol{U}}=P_{l}
$$

where $\boldsymbol{L}, \boldsymbol{M}$ are operators of elastic and mass forces of the shell; $\boldsymbol{U}=\left(u_{1}, u_{2}, w\right)$ is the displacement vector, $P_{l}$ is the liquid pressure.

Hereinafter we denote the surface of an empty tank as $S$. To define the pressure on the wetted parts of the tank we need the velocities of liquid level changing in each compartment. Let these velocities will be $V_{I}(t)$ and $V_{2}(t)$ respectively in first and second compartments. The domains occupied with liquid we denote as $\Omega_{1}$ и $\Omega_{2}$ for first and second compartments. The liquid densities will be $\rho_{1}$ and $\rho_{2}$ respectively. Filling levels in compartments will be denoted as $h_{1}$ and $h_{2}$. It would be noted that velocity potential function $\Phi$ is satisfied to Laplace equation. The components of dynamical pressure on tank walls in compartments are defined as

$$
p_{1}=-\rho_{1}\left(\frac{\partial \Phi}{\partial t}+V_{1}(t) \frac{\partial \Phi}{\partial z}\right) ; \quad p_{2}=-\rho_{2}\left(\frac{\partial \Phi}{\partial t}+V_{2}(t) \frac{\partial \Phi}{\partial z}\right) .
$$

For velocity potential $\Phi$ we obtain the mixed boundary value problem for Laplace equation in double domain $\Omega_{1} \cup \Omega_{2}$. Hereinafter we denote the normal displacement component of tank structure as $w$. 
Then the kinematical boundary condition of the continuous fluid motion on the wetted shell surface $S$ can be represented as follows:

$$
\frac{\partial \Phi}{\partial n}=\frac{\partial w}{\partial t}
$$

where $n$ is an external unit normal to wetted surface.

Let functions $\varsigma_{1}(t, x, y, z)$ and $\varsigma_{2}(t, x, y, z)$ describe the shapes and positions of free surfaces. These surfaces are denoted as $S_{10}, S_{20}$ in Fig. 1. On free surfaces the following formulae for pressure components are valid

$$
p_{1}-p_{10}=-\rho_{1}\left(\frac{\partial \Phi}{\partial t}+V_{1}(t) \frac{\partial \Phi}{\partial z}+g \zeta_{1}\right) ; \quad p_{2}-p_{20}=-\rho_{2}\left(\frac{\partial \Phi}{\partial t}+V_{2}(t) \frac{\partial \Phi}{\partial z}+g \zeta_{2}\right) \text {. }
$$

Here $\mathrm{g}$ - the gravity acceleration.

To determine the function $\Phi$ the following boundary value problem in the double domain $\Omega_{1} \cup \Omega_{2}$ is formulated with free-surface boundary conditions (kinematical and dynamical) and non-penetration condition on wetted parts:

$$
\begin{array}{r}
\Delta \Phi=\frac{\partial^{2} \Phi}{\partial x^{2}}+\frac{\partial^{2} \Phi}{\partial y^{2}}+\frac{\partial^{2} \Phi}{\partial z^{2}}=0 ;\left.\frac{\partial \Phi}{\partial n}\right|_{S}=\frac{\partial w}{\partial t} ;\left.\frac{\partial \Phi}{\partial n}\right|_{S_{10}}=\frac{\partial \zeta_{1}}{\partial t} ;\left.\quad \frac{\partial \Phi}{\partial n}\right|_{S_{20}}=\frac{\partial \zeta_{2}}{\partial t} \\
\frac{\partial \Phi}{\partial t}+V_{1}(t) \frac{\partial \Phi}{\partial z}+\left.g \zeta_{1}\right|_{s_{10}}=0 ; \frac{\partial \Phi}{\partial t}+V_{2}(t) \frac{\partial \Phi}{\partial z}+\left.g \zeta_{2}\right|_{s_{20}}=0 .
\end{array}
$$

So it is necessary to solve equations (1) and (2) simultaneously including the boundary condition for shell structure and using the next presentation for the liquid pressure on tank walls:

$$
p_{l}=\left(P_{l}, n\right)=\left\{\begin{array}{l}
-\rho_{1}\left(\frac{\partial \Phi}{\partial t}+V_{1}(t) \frac{\partial \Phi}{\partial z}\right) ; M \in \partial \Omega_{1}, \\
-\rho_{2}\left(\frac{\partial \Phi}{\partial t}+V_{2}(t) \frac{\partial \Phi}{\partial z}\right) ; M \in \partial \Omega_{2} .
\end{array}\right.
$$

\section{Mode superposition method}

We will seek the natural modes of vibration for double tank interacting with the fluid in the form

$$
\mathbf{u}=\sum_{k=1}^{N} c_{k} \mathbf{u}_{k}
$$


Here functions $\mathbf{u}_{k}(x, y, z)$ are natural vibration modes of the empty tank, $c_{k}(t)$ are unknown factors. The own modes of an empty tank are defined on the cylindrical part of shell structure, its bottom and baffle.

We will seek the function $\Phi_{1}$ as a sum of two potentials $\Phi=\Phi_{1}+\Phi_{2}$. To determine $\Phi_{1}$ we use the series

$$
\Phi_{1}=\sum_{k=1}^{N} \dot{c}_{k} \varphi_{1 k}
$$

Here factors $c_{k}(t)$ were defined in (3). To determine $\varphi_{1 k}$ we have the following boundary value problems:

$$
\nabla^{2} \varphi_{1 k}=0 ;\left.\quad \frac{\partial \varphi_{1 k}}{\partial n}\right|_{S_{1}}=w_{k} ;\left.\quad \varphi_{1 k}\right|_{S_{10}}=0 ;\left.\quad \varphi_{1 k}\right|_{S_{20}}=0
$$

It follows from (4) that the problem for double domain is reduced to boundary value problems for two single domains

$$
\nabla^{2} \varphi_{1 k}^{i}=0 ; \quad M \in \Omega_{i} ;\left.\quad \frac{\partial \varphi_{1 k}^{i}}{\partial n}\right|_{S_{i}}=w_{k} ; \quad M \in \Omega_{i} ;\left.\varphi_{1 k}^{i}\right|_{S_{i 0}}=0 .
$$

Here $S=S_{1}+S_{2} ; S_{1}, S_{2}$ are wetted parts of double tank surface.

Then for potential $\Phi_{1}$ we obtain the next representation:

$$
\Phi_{1}=\sum_{k=1}^{N} \dot{c}_{k} \varphi_{1 k}^{i}, \quad M \in \partial \Omega_{i} ; \quad i=1,2
$$

Before determining the function $\Phi_{2}$ let us consider the auxiliary problems for two fluid-filled compartments.

$$
\nabla^{2} \Psi_{i}=0 ;\left.\quad \frac{\partial \Psi_{i}}{\partial \mathbf{n}}\right|_{S_{i}}=0 ;\left.\quad \frac{\partial \Psi_{i}}{\partial n}\right|_{S_{i 0}}=\frac{\partial \zeta_{i}}{\partial t} ; \quad \frac{\partial \Psi_{i}}{\partial t}+\left.g \zeta_{i}\right|_{S_{i 0}}=0
$$

Considering harmonic vibrations and omitting indexes, we supposed $\Psi=\psi e^{i \chi t}$. So we have the eigenvalue problems in each compartment. Let denote as $\phi_{2 k}^{1}, \chi_{k}^{1}, \phi_{2 k}^{2}, \chi_{k}^{2}$ the modes and frequencies for first and second compartment respectively. The following relations are valid on free surfaces of compartment:

$$
\frac{\partial \varphi_{2 k}^{1}}{\partial n}=\left.\frac{\left(\chi_{k}^{1}\right)^{2}}{g} \varphi_{2 k}^{1}\right|_{S_{10}} ; \quad \frac{\partial \varphi_{2 k}^{2}}{\partial n}=\left.\frac{\left(\chi_{k}^{2}\right)^{2}}{g} \varphi_{2 k}^{2}\right|_{S_{20}}
$$


Then the potential $\Phi_{2}$ will be represented in the form

$$
\Phi_{2}= \begin{cases}\sum_{k=1}^{M_{1}} \dot{b}_{k} \varphi_{2 k}^{1}, & M \in \partial \Omega_{1} \\ \sum_{k=1}^{M_{1}} \dot{d}_{k} \varphi_{2 k}^{2}, & M \in \partial \Omega_{2}\end{cases}
$$

Accordingly, functions $\varsigma_{1}(t, x, y, z)$ and $\varsigma_{2}(t, x, y, z)$ can be written as

$$
\zeta_{1}=\sum_{k=1}^{N} c_{k} \frac{\partial \varphi_{1 k}^{1}}{\partial n}+\sum_{k=1}^{M} b_{k} \frac{\partial \varphi_{2 k}^{1}}{\partial n} ; \quad \zeta_{2}=\sum_{k=1}^{N} c_{k} \frac{\partial \varphi_{1 k}^{2}}{\partial n}+\sum_{k=1}^{M} d_{k} \frac{\partial \varphi_{2 k}^{2}}{\partial n}
$$

The velocity potential $\Phi$ took the form

$$
\Phi= \begin{cases}\sum_{k=1}^{N} \dot{c}_{k} \varphi_{1 k}^{1}+\sum_{k=1}^{M_{1}} \dot{b}_{k} \varphi_{2 k}^{1}, & M \in \Omega_{1} \\ \sum_{k=1}^{N} \dot{c}_{k} \varphi_{1 k}^{1}+\sum_{k=1}^{M_{1}} \dot{d}_{k} \varphi_{2 k}^{2}, & M \in \Omega_{2}\end{cases}
$$

To determine the pressure we have the next relationship:

$$
p_{l}=\left\{\begin{array}{l}
-\rho_{1}\left(\sum_{k=1}^{N} \ddot{c}_{k} \varphi_{1 k}^{1}+\sum_{k=1}^{M_{1}} \ddot{b}_{k} \varphi_{2 k}^{1}+V_{1}(t) \sum_{k=1}^{N} \dot{c}_{k} \frac{\partial \varphi_{1 k}^{1}}{\partial n}+V_{1}(t) \sum_{k=1}^{N} \dot{b}_{k} \frac{\partial \varphi_{2 k}^{1}}{\partial n}\right) ; M \in \partial \Omega_{1}, \\
-\rho_{2}\left(\sum_{k=1}^{N} \ddot{c}_{k} \varphi_{1 k}^{2}+\sum_{k=1}^{M_{2}} \ddot{d}_{k} \varphi_{2 k}^{2}+V_{2}(t) \sum_{k=1}^{N} \dot{c}_{k} \frac{\partial \varphi_{1 k}^{2}}{\partial n}+V_{2}(t) \sum_{k=1}^{N} \dot{d}_{k} \frac{\partial \varphi_{2 k}^{2}}{\partial n}\right) ; M \in \partial \Omega_{2} .
\end{array}\right.
$$

Using these relations for $\Phi, p_{l}, \varsigma_{1}(t, x, y, z)$ and $\varsigma_{2}(t, x, y, z)$, substituting them into eqns (1) and (2), and performing dot products we have obtained the following system of ordinary differential equations

$$
\begin{aligned}
& c_{j}(t) \Omega_{j}^{2}+\ddot{c}_{j}(t)=-\rho_{2}\left(\sum_{k=1}^{N} \ddot{c}_{k}(t)\left(\varphi_{1 k}^{1}, \mathbf{u}_{j}^{1}\right)+\sum_{k=1}^{M_{1}} \ddot{b}_{k}(t)\left(\varphi_{2 k}^{1}, \mathbf{u}_{j}^{1}\right)\right)- \\
& -\rho_{2} V_{1}(t)\left[\sum_{k=1}^{N} \dot{c}_{k}(t)\left(\frac{\partial \varphi_{1 k}^{1}}{\partial z}, \mathbf{u}_{j}^{1}\right)+\sum_{k=1}^{M_{1}} \dot{b}_{k}(t)\left(\frac{\partial \varphi_{2 k}^{1}}{\partial z}, \mathbf{u}_{j}^{1}\right)\right]-
\end{aligned}
$$




$$
\begin{aligned}
& -\rho_{2}\left(\sum_{k=1}^{N} \ddot{c}_{k}(t)\left(\varphi_{1 k}^{2}, \mathbf{u}_{j}^{2}\right)+\sum_{k=1}^{M_{2}} \ddot{d}_{k}(t)\left(\varphi_{2 k}^{2}, \mathbf{u}_{j}^{2}\right)\right)- \\
& -\rho_{2} V_{2}(t)\left[\sum_{k=1}^{N} \dot{c}_{k}(t)\left(\frac{\partial \varphi_{1 k}^{2}}{\partial z}, \mathbf{u}_{j}^{2}\right)+\sum_{k=1}^{M_{2}} \dot{d}_{k}(t)\left(\frac{\partial \varphi_{2 k}^{2}}{\partial z}, \mathbf{u}_{j}^{2}\right)\right] ; \\
& \alpha_{1 j} \ddot{b}_{j}(t)+V_{1}(t)\left[\sum_{k=1}^{N} \dot{c}_{k}(t)\left(\frac{\partial \varphi_{1 k}^{1}}{\partial z}, \varphi_{2 j}^{1}\right)+\alpha_{1 j} \dot{b}_{j}(t) \frac{\left(\chi_{j}^{1}\right)^{2}}{g}\right]+ \\
& +g \sum_{k=1}^{N} c_{k}(t)\left(\frac{\partial \varphi_{1 k}^{1}}{\partial n}, \varphi_{2 j}^{1}\right)+\alpha_{1 j} b_{j}(t)\left(\chi_{j}^{1}\right)^{2}=0 ; j=1, \ldots, M_{1} .
\end{aligned}
$$

Here

$$
\alpha_{k j}=\left(\varphi_{2 j}^{k}, \varphi_{2 j}^{k}\right) ; \quad k=1,2 ; \quad \mathbf{u}_{j}= \begin{cases}\mathbf{u}_{j}^{1} ; & M \in S_{1} ; \\ \mathbf{u}_{j}^{2} ; & M \in S_{2} .\end{cases}
$$

At first we consider the problem without including the liquid level changing, i.e. supposing $V_{1}(t)=V_{2}(t)=0$.

We will seek the solution of system (6) in the form

$$
b_{k}(t)=B_{k} e^{i \omega t} ; \quad c_{k}(t)=C_{k} e^{i \omega t} ; \quad d_{k}(t)=D_{k} e^{i \omega t} .
$$

Then we obtain the next eigenvalue problem to define frequencies $\omega$ and modes $X=\left(C_{k}, B_{k}, D_{k}\right)^{T}$ :

$$
\left(-\omega^{2} M+G\right) X=0
$$

where

$$
\begin{gathered}
M=\left(\begin{array}{ccc}
E+\rho_{2} P & \rho_{2} B^{1} & \rho_{2} B^{2} \\
0 & E & 0 \\
0 & 0 & E
\end{array}\right) ; \quad G=\left(\begin{array}{ccc}
\Omega & 0 & 0 \\
g G^{1} & H_{1} & 0 \\
g G^{2} & 0 & H_{2}
\end{array}\right) ; \\
G^{i}=\left\{g_{k j}^{i}\right\}, \quad g_{k j}^{i}=\left(\frac{\partial \varphi_{1 k}^{i}}{\partial n}, \frac{\varphi_{2 j}^{i}}{\alpha_{i j}}\right) ; \quad k=1, M_{i} ; j=1, N \\
B^{i}=\left\{b_{j k}^{i}\right\}, \quad b_{j k}^{i}=\left(\varphi_{2 k}^{i}, \mathbf{u}_{j}^{i}\right) ; \quad j=1, . ., N ; k=1, \ldots, M_{i}
\end{gathered}
$$

Here $\Omega, H_{1}, H_{2}$ are diagonal matrixes with squares of frequencies as diagonal elements for free vibrations of empty tank, liquid sloshing in first and second tank compartments accordingly. 


\section{BEM solution of velocity potential $\Phi_{1}$}

The velocity potential $\varphi_{1 k}$ for the $k$-th eigen - frequency of tank oscillation - is governed by the Laplace equation

$$
\nabla^{2} \varphi_{1 k}=0
$$

with boundary conditions (4) where the wall normal displacement is $w=u_{z}$ on the bottom and $w=u_{r}$ on the cylindrical part of tank shell structure in cylindrical coordinate system.

In the framework of boundary element method the eqn (7) may be rewritten into following integral form in the following way [18]

$$
c\left(M_{0}\right) \varphi_{1 k}\left(M_{0}\right)+\int_{\Gamma} \varphi_{1 k}(M)\left(\vec{\nabla} u^{*}, \vec{n}\right) d S=\int_{\Gamma}\left(q_{k}, \vec{n}\right) u^{*} d S ;\left(q_{k}, \vec{n}\right)=\frac{\partial \varphi_{1 k}}{\partial n} ; \Gamma=S_{i} \cup S_{i 0}
$$

where $M_{0}$ is the source or collocation point, $\vec{n}$ is a unit normal to the boundary, pointed out of the domain and $u^{*}$ is the fundamental Laplace solution $u^{*}=\left(4 \pi \cdot\left|M-M_{0}\right|\right)^{-1} ; \quad c\left(M_{0}\right)$ is the geometrical factor defined as $c\left(M_{0}\right)=\alpha / 4 \pi$, where $\alpha$ is an inner angle with origin in collocation point $M_{0}$.

The boundary of the computational domain is discretized by boundary elements $\Gamma=\sum_{b} \Gamma_{b}$. Each boundary element consists of 9 nodes for quadratic interpolation of functions and 4 nodes for linear interpolation of flux. Velocity potential is interpolated over the boundary elements as $\phi_{1 k}=\sum L_{i} \phi_{k, i}$. Flux is interpolated over the boundary elements as $q_{k}=\sum L_{i}^{\prime} q_{k, i}$, using discontinuous linear interpolation scheme, avoiding the definition problem in corner points. By applying the described interpolation the following form of eqn (8) can be written as:

$$
c\left(M_{0}\right) \varphi_{1 k}\left(M_{0}\right)+\sum_{b} \int_{\Gamma_{b}} L_{i} \varphi_{k, i}\left(\vec{\nabla} u^{*}, \vec{n}\right) d S=\sum_{b} \int_{\Gamma_{b}}\left(L_{i}^{\prime} q_{k, i}, \vec{n}\right) u^{*} d S
$$

with $i$ denoting the node number. After the following integral are calculated,

$$
[H]=\int_{\Gamma} L_{i}\left(\vec{\nabla} u^{*}, \vec{n}\right) d S ;[G]=\int_{\Gamma} L_{i}^{\prime} u^{*} d S
$$

the eqn (8) is transformed into the matrix form

$$
c\left(M_{0}\right) \varphi_{1 k}\left(M_{0}\right)+[H]\left\{\varphi_{1 k}\right\}=[G]\left\{q_{k}\right\} .
$$


The square brackets in eqn (9) denote integral matrixes and each source point yields one row in these matrixes. Gaussian quadrature algorithm was used for calculation the integrals, which were evaluated in local coordinate system. A weighted summation of up to 48 integration points on each coordinate axis was used. Free coefficient $c\left(M_{0}\right)$ is calculated indirectly. Rigid body movement $\phi=1, q=0$ is considered and thus the sum of all $[H]$ matrix elements for each source point is zero. This fact is considered in $c\left(M_{0}\right)$ calculations and these values are added to the diagonal terms of the $[H]$ matrix. After application of boundary conditions the system nay be solved for unknown boundary values of velocity potential or its normal derivative. The method is based on BEM and used the fluid flow solver developed by Ravnik et al. [19].

\section{Numerical results}

Let us consider the double tank with compartments partially filled with the fluid. The geometry of the tank is shown in Figure 1 and the parameters are following: the radius is $R=1.5 \mathrm{~m}$, the thickness is $h=0.01 \mathrm{~m}$, the length $L=6 \mathrm{~m}$, Young's modulus $E=2 \cdot 10^{5} \mathrm{MPa}$, Poisson's ratio $v=0.3$, the material's density is $\rho=7800$ $\mathrm{kg} / \mathrm{m}^{3}$, the fluid density in both compartments $\rho_{1}=\rho_{2}=1000 \mathrm{~kg} / \mathrm{m}^{3}$. The filling level of the fluid is denoted as $h$ (Fig. 1). It is equal in both compartment, its value $\mathrm{h}=1.5 \mathrm{~m}$. Boundary conditions are following: $u_{r}=u_{z}=u_{\theta}=0$ to $z=0$ and $r=R$.

The modes and frequencies of empty shell were obtained using FEM as it was described in [20]. The axisymmetric form was under consideration. The number of natural modes was equal to 20 . Here we used 20 elements along bottom and baffle and 20 elements along cylindrical part. Functions $\varphi_{1 k}$ were calculated by method developed in [19] and based on BEM. The simulation of top and bottom compartments was done separately. Boundary conditions were obtained by interpolation of structural analysis. The computational mesh (Fig. 1) had 3584 elements with 14338 function nodes and 14336 flux nodes.

The functions and frequencies $\phi_{2 k}^{1}, \chi_{k}^{1}, \phi_{2 k}^{2}, \chi_{k}^{2}$ of liquid vibration for this tank were calculated analytically and can be represented in the form

$$
\chi_{k}^{2}=g \frac{\mu_{k}}{R} \tanh \left(\frac{\mu_{k}}{R} h\right)_{;} \varphi_{2 k}=J_{0}\left(\frac{\mu_{k}}{R_{0}} r\right) \operatorname{ch}\left(\frac{\mu_{k}}{R_{0}} z\right) ; \alpha_{k}=\operatorname{ch}\left(\frac{\mu_{k}}{R_{0}} H\right)\left\|J_{0}\left(\frac{\mu_{k}}{R_{0}} r\right)\right\|
$$

for both departments. The numbers of own liquid modes were $M_{1}=M_{2}=20$ for both compartments. Fig 2 demonstrates modes of liquid vibration corresponding to $1^{\text {st }}, 2^{\text {nd }}, 8^{\text {th }}$ and $10^{\text {th }}$ modes of the liquid in the double tank. The results were obtained by method [19]. 


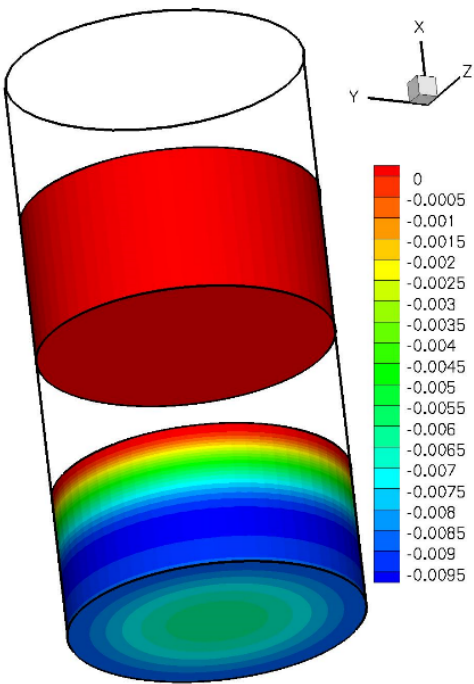

Mode 1

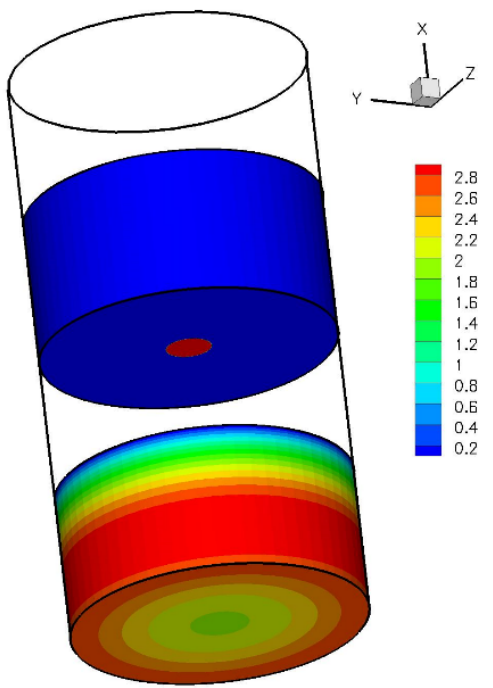

Mode 8

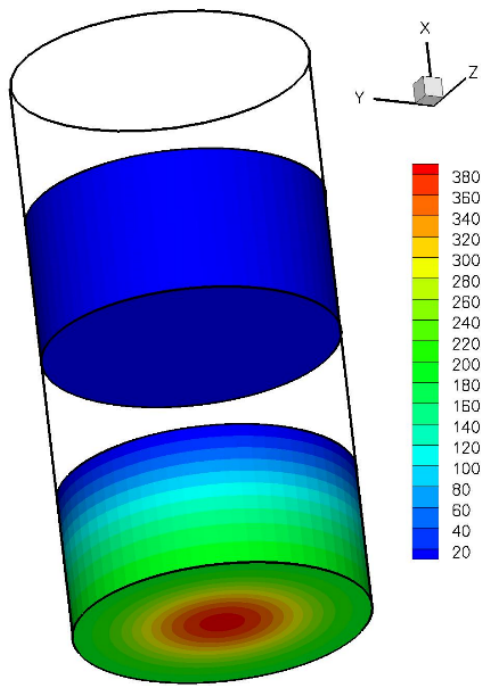

Mode 2

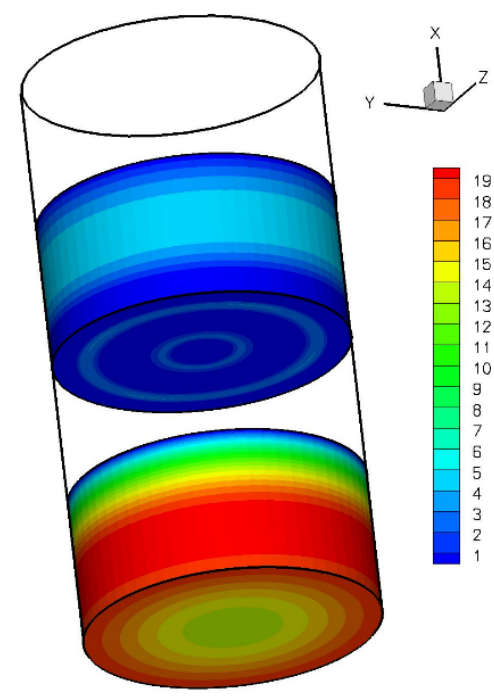

Mode 10

Figure 2: Modes of vibrations of double tank.

Table 1 provides the numerical values of the natural frequencies of vibration for an empty and fluid-filled double tank. Here coefficients $C_{1}, C_{2}, B_{1}, B_{2}$ indicate the mode of vibration. Coefficients $C_{1}, C_{2}$ are regarded to shell walls vibrations in first and second compartments, and coefficients $B_{1}, B_{2}$ corresponds to modes of liquid sloshing in first and second compartments accordingly. 
Table 1: Frequencies of the double tank vibrations.

\begin{tabular}{|c|c|c|c|c|c|c|}
\hline $\mathrm{n}$ & $C_{1}$ & $C_{2}$ & $B_{1}$ & $B_{2}$ & Empty tank & Fluid-filled tank \\
\hline 1 & 0.01 & 0.00 & 0.97 & 0.00 & & 5.00103 \\
\hline 2 & 0.00 & 0.01 & 0.00 & 0.97 & & 5.00173 \\
\hline 3 & 0.01 & 0.00 & 0.97 & 0.00 & & 6.77016 \\
\hline 4 & 0.00 & 0.02 & 0.00 & 0.97 & & 6.78022 \\
\hline 5 & 0.82 & 0.54 & 0.21 & 0.01 & 33.4493 & 7.03487 \\
\hline 6 & 0.28 & 0.85 & 0.01 & 0.31 & 34.4767 & 7.29735 \\
\hline 7 & 0.06 & 0.00 & 0.95 & 0.00 & & 8.15270 \\
\hline 8 & 0.00 & 0.08 & 0.00 & 0.947 & & 8.15270 \\
\hline 23 & 0.82 & 0.54 & 0.23 & 0.01 & 130.329 & 39.39860 \\
\hline 24 & 0.51 & 0.85 & 0.01 & 0.31 & 134.221 & 40.76115 \\
\hline 25 & 0.91 & 0.2 & 0.07 & 0.01 & 292.025 & 107.45330 \\
\hline 26 & 0.2 & 0.947 & 0.028 & 0.08 & 300.718 & 110.89764 \\
\hline 27 & 0.82 & 0.50 & 0.21 & 0.01 & 516.812 & 223.56725 \\
\hline 28 & 0.52 & 0.85 & 0.01 & 0.31 & 533.886 & 224.72695 \\
\hline
\end{tabular}

\section{Conclusions}

The numerical procedure based on a coupling finite element formulation and the boundary element method is developed for numerical analysis of fluid-structure interaction for a double tank. We introduce the representation of the velocity potential as the sum of two potential, one of them corresponds to problem of the fluid free vibrations in the rigid shell and another one corresponds to problem of elastic shell with fluid without including the gravitational component. Integration by the fluid volume is accomplished using BEM based fluid flow solver. The spectrum of frequencies for double tank was analysed.

\section{Acknowledgements}

The authors gratefully acknowledge Professor Carlos Brebbia for his constant support and interest in our research. We are also grateful to Ukrainian and Slovenian Ministries for our research grant "Development of fast boundary element method for fluid flow applications".

\section{References}

[1] R.A. Ibrahim, Liquid Sloshing Dynamics, Cambridge University Press, Cambridge, 2005.

[2] Sidi, M. J. Spacecraft Dynamics and Control, Cambridge University Press, New York, 1997.

[3] Robinson, H.G.R. and C.R. Hume. "Europa I: Flight Trial of F1", June 05, 1964.

[4] Space Exploration Technologies Corp. Demo Flight 2 Flight Review Update, June 15, 2007. 
[5] Vreeburg, Jan P.B. Spacecraft Maneuvers and Slosh Control, in IEEE Control Systems Magazine, June 2005.

[6] Biswal, K. C., Bhattacharyya, S. K. \& Sinha, P. K. Dynamic characteristics of liquid filled rectangular tank with baffles, IE (I) Journal-CV 84: pp. 145-148, 2004.

[7] Ranganathan, R., Ying, Y., and Miles, J., Analysis of Fluid Slosh in Partially Filled Tanks and Their Impact on the Directional Response of Tank Vehicles, SAE Technical Paper, No. 10, pp. 932-942, 1993.

[8] L. Strandberg, Lateral Stability of Road Tankers, VTI Report No. 138A, vol. 1, 1978.

[9] J.A. Romero, R. Hildebrand, M. Martínez, O. Ramírez, J.M. Fortanell, Natural sloshing frequencies of liquid cargo in road tankers, Int. J. Heavy Veh. Syst. 12, pp. 121-138, 2005.

[10] L. Xu, Fluid Dynamics in Horizontal Cylindrical Containers and Liquid Cargo Vehicle Dynamics, Ph.D. Thesis, University of Regina, Saskatchewan, 2005.

[11] S.M. Hasheminejad, M. Aghabeigi, Liquid sloshing in half-full horizontal elliptical tanks, J. Sound Vib. 324, pp. 332-349, 2009.

[12] A.M. Silveira, D.G. Stephens, H.W. Leonard, An Experimental Investigation of Liquid Oscillation in Tanks with Various Baffles, NASA Technical Note D-715, 1961.

[13] H.N. Abramson, Slosh Suppression, NASA Technical Report SP-8031, 1969.

[14] D.V. Evans, P. McIver, Resonant frequencies in a container with a vertical baffle, J. Fluid Mech. 175, pp. 295-307, 1987.

[15] E.B.B. Watson, D.V. Evans, Resonant frequencies of a fluid container with internal bodies, J. Eng. Math. 25, pp. 115-135, 1991.

[16] V. Armenio, M.L. Rocca, On the analysis of sloshing of water in rectangular containers: numerical study and experimental validation, Ocean Eng. 23, pp. 705-739, 1996.

[17] K. Modaressi-Tehrani, S. Rakheja, I. Stiharu, Three-dimensional analysis of transient slosh within a partly-filled tank equipped with baffles, Veh. Syst.Dyn. 45, pp. 525-548, 2007.

[18] Brebbia, C.A., Telles, J.C.F. \& Wrobel, L.C. Boundary Element Techniques, Springer-Verlag: Berlin and New York, 1984.

[19] Ravnik J., Škerglet L. and Z. Žunic. Velocity-vorticity formulation for 3D natural convection in an inclined enclosure by BEM. Int. J. Heat Mass Transfer, 51, pp. 4517-4527, 2008.

[20] Ventsel E., Naumenko V., Strelnikova E., Yeseleva E. Free vibrations of shells of revolution filled with a fluid. Engineering analysis with boundary elements, 34, pp. 856-862, 2010. 